\title{
Dynamics of the IL-33/ST2 network in the progression of human colorectal adenoma to sporadic colorectal cancer
}

Guanglin Cui ${ }^{1,2,3}$, Haili Qi ${ }^{1}$, Mona D. Gundersen ${ }^{3}$, Hang Yang ${ }^{1}$, Ingrid Christiansen ${ }^{3}$, Sveinung W. Sørbye ${ }^{4}$, Rasmus Goll ${ }^{3}$, Jon Florholmen ${ }^{3}$

${ }^{1}$ Department of Gastroenterology, the Second Affiliated Hospital of Zhengzhou University, Zhengzhou, Henan, China

${ }^{2}$ Faculty of Health, Nord-Trøndelag University College, Levanger, Norway

${ }^{3}$ Research Group of Gastroenterology \& Nutrition, ${ }^{4}$ Department of Clinical Pathology, Institute of Clinical Medicine, Faculty of Medicine, University of Troms $\varnothing$, Troms $\emptyset$, Norway

Correspondence to: Guanglin Cui, Department of Gastroenterology, the Second Affiliated Hospital of Zhengzhou University, 2 Jingba Road, Zhengzhou 455000, Henan, China E-mail: guanglin.cui@hint.no

Fax: +8663939084

Acknowledgments: This research was supported by grants from the Medical Research Program of Northern Norway Regional Health Authority (Grant No. SFP-44-04) and the National Natural Science Foundation of China (Grant No. 81071969). 
Abbreviations:

CRC: colorectal cancer

CT: cycle threshold

IBD: inflammatory bowel diseases

IFN: interferon

IHC: immunohistochemistry

IL: interleukin

LGD: lower grade dysplasia

HGD: high grade dysplasia

HPF: high-power fields

MGD: moderate grade dysplasia

MVD: microvessel densities

PCR: polymerase chain reaction

SEM: mean of standard error

Th: T helper

TNF: tumor necrosis factor

TNM: tumor/node/metastasis 


\section{Abstract}

Most sporadic colorectal cancers (CRCs) develop from preformed adenomas. Cytokines are involved in the transition from adenoma to CRC. Interleukin-33 (IL-33) is a newly discovered proinflammatory cytokine belonging to the IL-1 cytokine family and involved in the development of chronic inflammation and cancer. The aim of this study is to evaluate the dynamics of the IL-33/ST2 axis during the sequence of progression from normal colorectum to adenoma to carcinoma and to investigate the association of IL-33 and ST2 expression with clinicopathological parameters and prognosis. The results demonstrated that the levels of IL33 and ST2 in adenomas $(n=50)$, determined by real-time PCR, were significantly higher than those of normal controls $(n=30)$; the levels of both IL-33/ST mRNA in CRCs $(n=50)$ were higher than in normal controls but lower than levels in adenomas. Further analysis revealed that the expression level of ST2 in CRCs was associated with tumor/node/metastasis (TNM) stage. The log-rank test showed that neither the IL-33 nor the ST2 expression level was correlated with overall survival in patients with CRC. The increased expression of IL33/ST2 in adenomas and CRC tissues was confirmed by immunohistochemistry and was observed in both the tumor stromal cells and adenomatous/cancerous cells. Notably, increased densities of IL-33-positive and ST2-positive microvessels were found in the stroma of adenomas and CRCs. In conclusion, increased expression of the IL-33/ST2 axis along the colorectal adenoma-carcinoma sequence might be involved in the neoplastic transformation via the participation of this axis in the regulation of angiogenesis.

Key words: Adenoma-carcinoma transition; Colorectal cancer; Interleukin-33; ST2 


\section{Précis}

The IL-33/ST2 axis changes along the adenoma-carcinoma progression sequence, particularly in the tumor stromal cells, microvessels and adenomatous/cancerous epithelium. An activated IL-33/ST2 axis might thus contribute to neoplastic transformation. 


\section{Introduction}

Human colorectal cancer (CRC) is the third most frequent cancer worldwide. According to the classic genetic model for colorectal tumorigenesis described by Fearon and Vogelstein [1], most sporadic CRCs develop primarily from a pre-existing principal precursor - an adenomatous polyp - through a progressive accumulation of a number of genetic mutations and molecular alterations $[2,3]$. The transition from an adenoma to a sporadic CRC may take many years and invariably evokes a strong inflammatory response that includes inflammatory cell infiltration and pro-inflammatory cytokine secretion in the tumor microenvironment [46]. Because these inflammatory components construct an inflammatory microenvironment and then contribute to the initiation and progression of CRC [7-12], it is important to investigate the dynamic temporal changes of this tumor inflammatory microenvironment. Indeed, we and others have previously demonstrated that the colorectal neoplastic transformation leads to remarkable changes in cytokine profiles in the tumor microenvironment $[7,9,13,14]$, which might have a significance in predicting the progression from adenoma to CRC and the prognosis of the CRC $[8,10,11,13-16]$. These findings provide new insights into transitional mechanisms of tumor progression and help to design novel interventional strategies.

Interleukin (IL)-33 is a newly discovered pro-inflammatory cytokine belonging to the IL-1 family. The IL-33/ST2 (IL-33 receptor) axis plays an important role in regulating both T helper (Th)1 and Th2 cell responses [17] and is involved in the pathogenesis of human inflammatory diseases $[18,19]$. For colorectal diseases, extensive evidence now suggests that IL-33 is activated in the inflamed mucosa of ulcerative colitis and is known to be correlated with the development of chronic inflammation $[18,20]$. Because the involvement of chronic inflammation, as seen in inflammatory bowel disease (IBD), has been suggested to be a 
critical mechanism underlying CRC, the role of IL-33/ST2 in tumors has recently attracted much attention and investigation. Studies have shown that the IL-33/ST2 pathway plays an active role in inhibiting antitumor immunity and subsequently promoting metastasis in breast cancer $[19,21,22]$. In inflammation-associated pancreatic cancer, it might function as a crucial mediator that stimulates the process of carcinogenesis [23]. Moreover, two studies have shown that IL-33 is associated with tumor cell invasion or metastasis in head and neck squamous cell carcinoma [24] and hepatocellular carcinoma [25]. The prognostic significance of IL-33 has also been examined. Several reports have revealed that cancer patients with a high expression of IL-33 have a poor prognosis [24, 26, 27]. However, the involvement of IL33 in different human tumors is controversial; a protective role of IL-33 in some types of human cancers has also been reported. For example, a reduced IL-33 plasma level in patients with multiple myeloma is associated with a more advanced stage of disease [28].

Despite extensive data describing the importance of IL-33/ST2 activation in the pathogenesis of chronic colorectal inflammation as seen in inflammatory bowel diseases $[18,20]$, the role of the IL-33/ST2 axis in the development of sporadic CRC has yet to be examined. It is known that the inflammatory response occurs throughout the adenoma-carcinoma sequence and contributes to the adenoma-cancer transition through the release of pro-tumor cytokines and other growth factors. In addition, we have previously found that the tumor microenvironment has a Th2 cytokine profile in the adenoma-carcinoma sequence [29]. Moreover, it has been shown that IL-33 enhances Th2 polarization [17]. Given this, we hypothesized that an altered IL-33/ST2 axis might be involved in the pathogenesis of colorectal neoplastic transformation. In the present study, therefore, we characterized the dynamic changes of the IL-33/ST2 axis in the tumor microenvironment and evaluated its significance along the colorectal adenoma-carcinoma sequence. 


\section{Patients and Methods}

\section{Patients}

Patients were enrolled at the Departments of Gastrointestinal Surgery and Gastroenterology at the University Hospital of North Norway: 50 colorectal adenomas excised completely by endoscopic polypectomy in patients ages 43-92 years, 50 CRC lesions excised by surgery in patients ages 42-89 years, and 30 morphologically normal colorectal mucosa samples confirmed by colonoscopic and histological examination in patients ages 24-79 years. Patients were recruited between August 2003 and December 2008 (for detailed information, refer to Table 1). None of the included patients or control subjects had a history of regular use of immunomodulation treatment or chemotherapy. All biopsies were prepared and routinely embedded in paraffin. Sections $(4 \mu \mathrm{m})$ were cut and stained with hematoxylin and eosin $(H \& E)$. Histological diagnosis of all biopsies was determined by experienced pathologists from the Department of Clinical Pathology at the University Hospital of North Norway and were reviewed by senior pathologist SWS. The study was approved by the Regional Ethical Committee of Northern Norway, and written informed consent was obtained from all patients.

\section{Real-time polymerase chain reaction (PCR) quantification of IL-33 and ST2 mRNA}

Biopsies were collected in RNAlater solution (Ambion Europe, Cambridgeshire, UK), total RNA was extracted by the TRIzol method (Invitrogen Life Tech., Carlsbad, CA, USA), and reverse transcription was performed with SuperScript II (Invitrogen Life Tech., Carlsbad, CA, USA). Real-time PCR was performed on an ABI-prism 7900 sequence detector with a TaqMan Gold ${ }^{\mathrm{TM}}$ PCR core reagents kit (Applied Biosystems/Roche, Branchburg, NJ, USA) in $25 \mu \mathrm{L}$ volume according to our previously published method [30]. The TaqMan primer sequences for the housekeeping gene (beta-actin) were: forward primer 5, TGCCGACAGGATGCAGAAG 3'; reverse primer 5' GCCGATCCACACGGAGTACT 3'; 
and probe FAM 5' AGATCAAGATCATTGCTCCTCCTGAGCGC 3' TAMRA. Primer sequences for IL-33 were: forward primer 5' TGAGTCTCAACACCCCTCAAATG 3'; reverse primer 5' GGCATGCAACCAGAAGTCTTTT 3'; and probe FAM 5', CAGGTGACGGTGTtGATGGTAAGATGTTAATG 3' BHQ. IL-33 receptor ST2 primers were purchased from Life Technologies (Cat\# No. 4331182; Grand Island, NY, USA). The expression of IL-33 and ST2 mRNA in tissues obtained from the colorectal adenomas and CRCs was measured by cycle threshold (CT) value relative to that of the normal control mucosa as fold difference $(\mathrm{N})=2^{-\Delta \Delta \mathrm{CT}}, \Delta \mathrm{CT}=\mathrm{CT}$ measured gene (IL-33 or ST2) $-\mathrm{CT}$ beta-actin, $\Delta \Delta \mathrm{CT}=$ $\Delta \mathrm{CT}$ adenoma or $\mathrm{CRC}$ - average $\Delta \mathrm{CT}$ normal as described in our recent publication [14]. The difference among the normal control, colorectal adenoma and CRC were compared by $\triangle \mathrm{CT}$ values.

Immunohistochemical examinations of IL-33-positive cells and ST2-positive cells in the tumor microenvironment

To evaluate the distribution and expression patterns of the IL-33/ST2 axis in the tumor microenvironment, immunohistochemistry (IHC) was performed using $4 \mu \mathrm{m}$ paraffin sections from the controls, adenomas and CRCs with a Vectastatin Elite ABC Kit (Vector Lab., Burlingame, CA, USA) according to the manufacturer's instructions and our published methods [31]. The following primary antibodies were used: goat anti-human IL-33 polyclonal antibody (working dilution 1:100; R\&D systems, Minneapolis, MN, USA) and mouse antihuman ST2 monoclonal antibody (working dilution 1:100; Medical \& Biological Laboratories Co. Ltd., Nagoya, Japan). Antibodies were incubated at $4^{\circ} \mathrm{C}$ overnight. 3-Amino-9ethylcarbazole (AEC; Vector Laboratories, Burlingame, CA, USA) was used as chromogen, and slides were slightly counterstained with Mayer's hematoxylin. Negative control slides for IHC were used routinely as follows: (1) primary antibodies were substituted with the isotype- 
matched control antibodies; (2) secondary antibody was substituted with phosphate buffered saline (PBS).

Evaluation of IL-33-expressing cell phenotypes in the tumor stroma by double IHC staining

To define the phenotypes of IL-33-expressing cells in the tumor microenvironment, double IHC staining with the antibodies IL-33 (rabbit polyclonal antibody from Abcam, UK)/CD34 (monoclonal antibody from Dako Cor., Carpinteria, CA, USA, to label microvessels), and IL33/SMA-alpha (monoclonal antibody from Dako Cor., Carpinteria, CA, USA, to label myofibroblasts) was performed using the EnVision Doublestain System kit (Dako Cor., Carpinteria, CA, USA) as previously described in our publication [14]. The 3-amino-9ethylcarbazole substrate kit for peroxidase (AEC, Vector Laboratories, Burlingame, CA, USA) was used for the visualization of IL-33 immunoreactivity, and the Vector Blue Alkaline Phosphatase Substrate Kit III (Vector Blue, Vector Laboratories, Burlingame, CA, USA) was used for CD34 and SMA-alpha immunoreactivity, respectively. Nuclear counterstaining was not applied.

\section{Morphometric analysis}

All stained slides were examined under light microscopy. IL-33-positive and ST2-positive cells were found to be present in both the lamina propria and the epithelium, and the densities of positive cells were evaluated in the lamina propria and epithelium according to the methods described in our previous publication [13]. In the stroma, semi-quantified scoring was performed in at least 3 optional fields with abundant distribution from each slide under $\times 400$ high-power fields (HPF) and was scored as: nil (0), 1-19 cells/field (1+), 20-49 cells/field (2+) and over 50 cells/field (3+). IL-33-positive and ST2-positive microvessel densities (MVDs) in the stroma were quantified according to the previously published method [32]. In the epithelium, IL-33-positive cells and ST2-positive cells were graded on a scale of 0-3, with 0 
representing no detectable staining and $3+$ representing the strongest staining. The average values were used for statistical analysis.

\section{Statistical analysis}

The results were expressed as the mean \pm SEM (mean of standard error) unless otherwise stated. Statistical significance was evaluated by the Mann-Whitney test or the Kruskal-Wallis test. Kaplan-Meier analysis was used to calculate survival rates and differences in survival curves were determined by the log-rank test. The Cox proportional hazards regression model with a stepwise procedure was used to analyze the simultaneous influence of prognostic factors. Values of $\mathrm{P}<0.05$ and $<0.01$ were considered significant. 


\section{Results}

On the transcript level, the expression of the IL-33/ST2 axis was dynamically altered along the adenomas-carcinoma sequence

The expression level of IL-33 mRNA in the adenoma tissues determined by real-time PCR was significantly increased compared to the normal controls (Fig. 1A). The expression of IL33 mRNA in the CRC tissues was also significantly higher than in the normal controls but lower than that in the adenomas (Fig. 1A).

The expression level of ST2 mRNA along the adenoma-carcinoma sequence was also changed. As seen in Fig. 1B, the change in ST2 expression showed a pattern similar to that of IL-33 expression but to a more moderate degree. ST2 expression was greatly increased in the adenoma tissues but not changed in the CRC tissues compared to the normal controls (Fig. 1B).

The expression levels of IL-33/ST2 mRNA were analyzed against clinical pathological parameters in adenomas and sporadic CRCs

Because the IL-33/ST2 axis has been reported to be involved in the pathogenesis of human cancers $[21,26,33]$, the expression levels of IL-33/ST2 mRNA were analyzed against clinical pathological parameters of adenomas and CRCs.

At the adenoma stage, the expression levels of both IL-33 and ST2 mRNAs were not correlated with degree of dysplasia (low grade dysplasia vs. moderate grade dysplasia $v s$. high grade dysplasia; IL-33: $6.74 \pm 1.69$ vs. $10.46 \pm 2.65$ vs. $11.71 \pm 8.65 ;$ ST2: $2.56 \pm 0.94$ vs. $4.09 \pm 1.81$ vs. $1.05 \pm 0.35$; both $\mathrm{P}>0.05$ by the Kruskal-Wallis test). IL-33 and ST2 mRNA levels also did not differ between different histological subtypes/groups (tubular vs. 
tubulovillous+villous; IL-33: $10.04 \pm 2.33$ vs. $6.99 \pm 2.05$; ST2: $3.40 \pm 1.50$ vs. $2.01 \pm 0.34$; both $\mathrm{P}>0.05$ by the Mann-Whitney test).

At the colorectal cancer stage, the expression level of IL-33 mRNA was not associated with TNM (tumor/node/metastasis) stage (TNM stage I + II vs. III+VI: $5.61 \pm 2.00$ vs. $4.89 \pm 2.65$ : P $>0.05$ by the Mann-Whitney test) or node involvement (node negative vs. node positive: $8.62 \pm 2.85$ vs. $8.01 \pm 4.72, \mathrm{P}>0.05$ by the Mann-Whitney test). Similarly, the expression level of ST2 mRNA was not associated with node involvement (node negative vs. node positive: $2.03 \pm 0.59$ vs. $3.75 \pm 1.09, \mathrm{P}>0.05$ by the Mann-Whitney test). However, the level of ST2 in CRC patients with advanced TNM stages was significantly higher than in those with early stages (TNM stage I + II vs. III+VI: $1.96 \pm 0.66$ vs. $3.59 \pm 0.95: \mathrm{P}<0.05$ by the Mann-Whitney test).

The significance of IL-33 and ST2 mRNA levels in predicating overall survival of patients with $C R C$

Previous studies have shown that the expression level of IL-33/ST2 in certain types of human cancers might predicate the survival time $[24,26,27]$. Therefore, we analyzed the relationship between the expression levels of IL-33/ST2 and clinical prognosis in patients with sporadic CRC. The overall survival data were available for 28 patients with CRC. Kaplan-Meier analysis revealed that both IL-33 level (Fig. 2A) and ST2 level (Fig. 2B) in cancer tissues were not associated with the overall survival in patients with CRC.

Immunohistochemistry of IL-33 and ST2 distribution patterns in the tumor microenvironment of adenomas and CRCs

Subsequently, we used IHC to examine the distribution patterns of IL-33 and its receptor, ST2, in the adenomatous and cancerous tumor microenvironments. 
In the normal controls, IL-33 immunoreactivity was observed in the lamina propria but rarely in the epithelium (Fig. 3A). In adenomas and CRCs, IL-33 immunoreactivity was observed in both the tumor stroma and the epithelium. Many IL-33-positive cells were located in tumorassociated microvessels (arrow in Fig. 3B \& C) and in adenomatous and cancerous epithelium (arrow in Fig. 3B and inserted image in Fig. 3C). The semi-quantitative grading scores of IL33-positive cells in the adenomatous epithelium (Fig. 4A) and tumor stroma (Fig. 4B) were significantly higher than in the normal control; the grading scores of IL-33 in the CRC epithelium and tumor stroma were also higher than in the normal control, but lower than in the adenomatous epithelium and stroma (Fig. 4A \& B).

ST2 immunoreactivity was observed in both the epithelium and lamina propria in normal controls (Fig. 3D), adenomas (Fig. 3E) and CRCs (Fig. 3F). Notably, some of tumorassociated microvessels in the adenoma/CRC tumor stroma (arrow in Fig. 3E for adenoma; arrow in Fig. 3E for CRC) were also positive for ST2 immunoreactivity. The semiquantitative grading score of ST2 immunoreactivity in the epithelium in all three groups was not significant different (see Fig. 4C). However, the grading score of ST2-positive stromal cells showed an increasing trend from the control to the adenoma stage and to the CRC stage (Fig. 4D).

Because recent studies have demonstrated that vascular endothelial cells are one of the dominant IL-33-expressing cell populations [34], and because we observed an increased IL33 or ST2 positive microvessels in the tumor microenvironment, we quantified the IL-33positive microvessel density (MVD) and ST2-positive MVD in the adenoma and the CRC tumor stroma. The results revealed that both IL-33-positive MVD (Fig. 4E) and ST2-positive MVD (Fig. 4F) were significantly increased in the adenoma stroma and non-significantly increased in the CRC stroma compared to normal controls. 
To further identify the phenotypes of IL-33-positive cells in the tumor stroma, double IHC using IL-33/CD34 (to mark tumor-associated microvessels) and IL-33/SMA-alpha (to mark tumor-associated myofibroblasts) was performed. Compared to the control, the co-expression of IL-33 with CD34 (Fig. 5A-C) and SMA-alpha (Fig. 5D-F) in the stroma of adenomas/CRCs was frequently observed. This finding indicates that most IL-33-positive cells in the stroma of adenomas/CRCs were tumor-associated microvessels and myofibroblasts. 


\section{Discussion}

Accumulated evidence has suggested that inflammatory cytokines, including tumor necrosis factor (TNF)- $\alpha$, IL-6 and IL-17A, are involved in the pathogenesis of the adenomacarcinoma transition $[9,13,14,35]$. In this study, we found that the expression profile of the IL-33/ST2 axis within the tumor microenvironment was altered throughout the adenomacarcinoma sequence and might be involved in the process of CRC. In particular, IHC examination demonstrated that high IL-33/ST2 expression is likely to be partially produced by the tumor-associated microvessels, fueling an autocrine pathway involving IL-33/ST2 expression and angiogenesis and ultimately influencing the development of colorectal adenomas and CRCs.

IL-33 is a novel cytokine with a potential pro-inflammatory capacity. The importance of IL-33 in the development of the colorectal inflammatory disease ulcerative colitis has been addressed $[18,20]$. Recent studies have also revealed that the elevation of IL-33 is involved in the pathogenesis of pancreatic cancer [23], gastric cancer [27] and liver cancer [25, 36], and ST2 is expressed in both the tumor cells and stromal cells [23]. However, there is very limited information available regarding the change in the IL-33/ST2 axis during the development of CRC. To our best knowledge, the current study is the first of its kind to demonstrate a dynamic change in the IL-33/ST2 axis from colorectal adenomas to sporadic CRCs. The increase in IL-33 began at the adenoma stage and, although it declined slightly at the cancer stage compared to the adenoma stage, it was higher in both stages compared to controls. This finding might indicate that IL-33 is an early response inflammatory element and might serve as an alarm index for the colorectal neoplastic transformation. Although the exact mechanism for this expression pattern is currently unclear, it might be related to the changed expression patterns of IL-33 regulatory factors at different time periods during the 
colorectal adenoma-carcinoma sequence. For instance, cytokines such as interferon (IFN)- $\gamma$ and TNF- $\alpha$ are the well documented stimulators for IL-33 production [37-39], and we have previously demonstrated that the expression of IFN-gamma and TNF- $\alpha$ are remarkably elevated in adenoma tissues but reduced in CRC tissues [13]. This might partially explain the dynamic changes in IL-33 expression along the adenoma-carcinoma sequence. The expression of ST2 along the adenoma-carcinoma sequence was shown a pattern similar to that of IL-33; the expression level of ST2 mRNA was greatly increased at the adenoma stage but was unchanged from normal at the CRC stage. Taken together, our quantitative findings indicate that the greatest change in the IL-33/ST2 axis along the adenoma-carcinoma sequence occurred at the adenoma stage.

With respect to the relationship between IL-33/ST2 and clinicopathological parameters of patients with adenomas and CRCs, we have found that although the difference did not reach statistical significance, the increased IL-33 expression in the adenoma tissues was associated with degree of dysplasia. Because the progression of dysplastic severity in adenomas is associated with a high malignant potential [40], our observation might imply that IL-33 is a potential stimulating factor for the disease stage progression. The expression level of ST2, but not IL-33, in the CRC tissues was associated with TNM stage; patients with advanced stages had a higher ST2 level than those with early stages. This finding is consist with the Jovanovic and colleagues' data observed in breast cancer [33] and suggests that ST2 could be an potential modulator in CRC progression by increasing binding avidity for IL-33. Previous publications have also reported that the expression level of IL-33/ST2 is related to survival in patients with certain types of cancer [24, 26, 27]. However, we could not confirm this relationship between the expression levels of IL-33/ST2 and survival in patients with sporadic CRC. The overall survival was not influenced by either IL-33 or ST2. However, given that the number of patients with CRC and survival data in this study was rather small 
(only 28 patients), it is necessary to investigate the prognostic significance of IL-33/ST2 in a large scale study of patients with CRC.

The expression of IL-33 and the ST2 receptor has been studied in several types of digestive system cancers $[23,25,27,36]$ and has been found to be localized in various cell types within specific microenvironments [23]. Our IHC results clearly showed increased IL33 and ST2 immunoreactivity in the adenomatous/cancerous epithelium, supporting the possibility that the IL-33/ST2 axis is involved in regulating the transformation of colorectal epithelium. Our double IHC observations confirmed IL-33 immunoreactivity in myofibroblasts in the tumor stroma, which is consistent with previous findings from studies of patients with ulcerative colitis demonstrating that myofibroblasts are an important cellular source of IL-33 [18, 29, 41, 42]. In this study, our IHC observations of increased IL-33 immunoreactivity in CD34-positive microvessels in the adenoma/CRC tumor stroma confirmed that vascular endothelial cells are the dominant IL-33-expressing cell population [34]. It has been recently reported that the pro-tumor mechanism of IL-33/ST2 is via enhanced angiogenesis in the mouse breast cancer model [33] and is mediated by its receptor, ST2, expressed in the effecting cells $[19,43]$. In this study, we found that IL-33 was expressed in adenomatous/cancerous epithelium, tumor-associated myofibroblasts and microvessels and that ST2 was expressed in tumor-associated microvessels. By further quantifying IL-33-positive and ST2-positive MVDs, we found that both were significantly increased in adenoma/CRC tumor stroma. Our present findings might strongly support the hypothesis that increased expression of the IL-33/ST2 axis along the adenoma-carcinoma sequence enhances angiogenesis via paracrine and autocrine mechanisms. Because the expression of ST2 in adenomatous/cancerous epithelium has also been observed, a direct effect of IL-33 on transformed epithelial cells cannot be excluded, and further investigation is required. 
In conclusion, our data reveal a dynamic change in the IL-33/ST2 axis along the colorectal adenoma-carcinoma sequence in which IL-33 and ST2 are more significantly increased at the adenoma stage than at the CTC stage. The expression levels of IL-33 and ST2 were not correlated with overall survival in patients with CRCs. Immunohistochemical identification of IL-33/ST2 phenotypes in adenoma/CRC might suggest a mixed cellular source in which tumor-associated microvessels are an important source of IL-33 and possess ST2 receptors in the tumor stroma; this might imply an autocrine mechanism of angiogenesis stimulation during the process of CRC. Additional studies are needed to further refine the exact functions and mechanisms of IL-33/ST2 contributing to the pathogenesis of adenomas and CRCs. 
Table 1. Histological data of specimens from normal controls and patients with adenomas and colorectal cancer

\begin{tabular}{|c|c|c|c|c|c|c|c|c|}
\hline & \multicolumn{2}{|c|}{ Gender } & \multicolumn{3}{|c|}{ Pathology } & \multicolumn{3}{|c|}{ Dysplasia } \\
\hline & male & female & tubular & tubulovillous & villous & LGD & MGD & HGD \\
\hline Normal & 20 & 10 & & & & & & \\
\hline \multirow[t]{3}{*}{ Adenoma } & 31 & 19 & 33 & 15 & 2 & 22 & 24 & 4 \\
\hline & & & & & & \multicolumn{3}{|c|}{ TNM stage } \\
\hline & & & adenocarcinoma & mucinous & signet-ring & I & II & III+IV \\
\hline Cancer & 42 & 8 & 45 & 4 & 1 & 9 & 20 & 21 \\
\hline
\end{tabular}

LGD: lower grade dysplasia; MGD: moderate grade dysplasia; HGD: high grade dysplasia. 
Conflicts of interest

All authors declare no conflicts of interest. 


\section{References}

1. Fearon ER, Vogelstein B (1990) A genetic model for colorectal tumorigenesis. Cell 61:759-767

2. Leslie A, Carey FA, Pratt NR, Steele RJ (2002) The colorectal adenoma-carcinoma sequence. Br J Surg 89:845-860

3. Khosraviani K (1996) Colorectal adenoma-carcinoma sequence. Gut 39:342

4. Xie K (2001) Interleukin-8 and human cancer biology. Cytokine Growth Factor Rev 12:375-391

5. Cacev T, Radosevic S, Krizanac S, Kapitanovic S (2008) Influence of interleukin-8 and interleukin-10 on sporadic colon cancer development and progression. Carcinogenesis 29:1572-1580

6. Waugh DJ, Wilson C (2008) The interleukin-8 pathway in cancer. Clin Cancer Res 14:6735-6741

7. Fantini MC, Pallone F (2008) Cytokines: from gut inflammation to colorectal cancer. Curr Drug Targets 9:375-380

8. Choi JW, Liu H, Shin DH, Yu GI, Hwang JS, Kim ES, Yun JW (2013) Proteomic and cytokine plasma biomarkers for predicting progression from colorectal adenoma to carcinoma in human patients. Proteomics 13:2361-2374

9. Chung YC, Chang YF (2003) Significance of inflammatory cytokines in the progression of colorectal cancer. Hepatogastroenterology 50:1910-1913

10. Kang M, Edmundson P, Araujo-Perez F, McCoy AN, Galanko J, Keku TO (2013) Association of plasma endotoxin, inflammatory cytokines and risk of colorectal adenomas. BMC cancer 13:91

11. Kim S, Keku TO, Martin C, Galanko J, Woosley JT, Schroeder JC, Satia JA, Halabi S, Sandler RS (2008) Circulating levels of inflammatory cytokines and risk of colorectal adenomas. Cancer Res 68:323-328

12. Krzystek-Korpacka M, Diakowska D, Kapturkiewicz B, Bebenek M, Gamian A (2013) Profiles of circulating inflammatory cytokines in colorectal cancer (CRC), high cancer risk conditions, and health are distinct. Possible implications for CRC screening and surveillance. Cancer Lett 337:107-114

13. Cui G, Goll R, Olsen T, Steigen SE, Husebekk A, Vonen B, Florholmen J (2007) Reduced expression of microenvironmental Th1 cytokines accompanies adenomascarcinomas sequence of colorectum. Cancer Immunol Immunother 56:985-995

14. Cui G, Yuan A, Goll R, Vonen B, Florholmen J (2009) Dynamic changes of interleukin-8 network along the colorectal adenoma-carcinoma sequence. Cancer Immunol Immunother 58:1897-1905

15. Oliveira Frick V, Rubie C, Ghadjar P, Faust SK, Wagner M, Graber S, Schilling MK (2011) Changes in CXCL12/CXCR4-chemokine expression during onset of colorectal malignancies. Tumour Biol 32:189-196

16. Pellegrini P, Berghella AM, Contasta I, Del Beato T, Adorno D (2006) The study of a patient's immune system may prove to be a useful noninvasive tool for stage classification in colon cancer. Cancer Biother Radiopharm 21:443-467

17. Liew FY (2012) IL-33: a Janus cytokine. Ann Rheum Dis 71 Suppl 2:i101-104

18. Beltran CJ, Nunez LE, Diaz-Jimenez D, Farfan N, Candia E, Heine C, Lopez F, Gonzalez MJ, Quera R, Hermoso MA (2010) Characterization of the novel ST2/IL-33 system in patients with inflammatory bowel disease. Inflamm Bowel Dis 16:10971107 
19. Milovanovic M, Volarevic V, Radosavljevic G, Jovanovic I, Pejnovic N, Arsenijevic N, Lukic ML (2012) IL-33/ST2 axis in inflammation and immunopathology. Immunol Res 52:89-99

20. Kobori A, Yagi Y, Imaeda H, Ban H, Bamba S, Tsujikawa T, Saito Y, Fujiyama Y, Andoh A (2010) Interleukin-33 expression is specifically enhanced in inflamed mucosa of ulcerative colitis. J Gastroenterol 45:999-1007

21. Jovanovic IP, Pejnovic NN, Radosavljevic GD, Arsenijevic NN, Lukic ML (2012) IL33/ST2 axis in innate and acquired immunity to tumors. Oncoimmunology 1:229-231

22. Eiwegger T, Akdis CA (2011) IL-33 links tissue cells, dendritic cells and Th2 cell development in a mouse model of asthma. Eur J Immunol 41:1535-1538

23. Schmieder A, Multhoff G, Radons J (2012) Interleukin-33 acts as a pro-inflammatory cytokine and modulates its receptor gene expression in highly metastatic human pancreatic carcinoma cells. Cytokine 60:514-521

24. Chen SF, Nieh S, Jao SW, Wu MZ, Liu CL, Chang YC, Lin YS (2013) The paracrine effect of cancer-associated fibroblast-induced interleukin-33 regulates the invasiveness of head and neck squamous cell carcinoma. J Pathol 231:180-189

25. Zhang P, Liu XK, Chu Z, Ye JC, Li KL, Zhuang WL, Yang DJ, Jiang YF (2012) Detection of interleukin-33 in serum and carcinoma tissue from patients with hepatocellular carcinoma and its clinical implications. J Int Med Res 40:1654-1661

26. Hu LA, Fu Y, Zhang DN, Zhang J (2013) Serum IL-33 as a diagnostic and prognostic marker in non- small cell lung cancer. Asian Pac J Cancer Prev 14:2563-2566

27. Sun P, Ben Q, Tu S, Dong W, Qi X, Wu Y (2011) Serum interleukin-33 levels in patients with gastric cancer. Dig Dis Sci 56:3596-3601

28. Musolino C, Allegra A, Profita M, Alonci A, Saitta S, Russo S, Bonanno A, Innao V, Gangemi S (2013) Reduced IL-33 plasma levels in multiple myeloma patients are associated with more advanced stage of disease. Br J Heamatol 160:709-710

29. Seidelin JB, Rogler G, Nielsen OH (2011) A role for interleukin-33 in $\mathrm{T}(\mathrm{H}) 2$ polarized intestinal inflammation? Mucosal Immunol 4:496-502

30. Cui G, Olsen T, Christiansen I, Vonen B, Florholmen J, Goll R (2006) Improvement of real-time polymerase chain reaction for quantifying TNF-alpha mRNA expression in inflamed colorectal mucosa: an approach to optimize procedures for clinical use. Scand J Clin Lab Inv 66:249-259

31. Cui G, Koh TJ, Chen D, Zhao CM, Takaishi S, Dockray GJ, Varro A, Rogers AB, Fox JG, Wang TC (2004) Overexpression of glycine-extended gastrin inhibits parietal cell loss and atrophy in the mouse stomach. Cancer Res 64:8160-8166

32. Cui J, Xu G, Liu J, Pang Z, Florholmen J, Cui G (2013) The expression of non-mast histamine in tumor associated microvessels in human colorectal cancers. Pathol Oncol Res 19:311-316

33. Jovanovic IP, Pejnovic NN, Radosavljevic GD, Pantic JM, Milovanovic MZ, Arsenijevic NN, Lukic ML (2014) Interleukin-33/ST2 axis promotes breast cancer growth and metastases by facilitating intratumoral accumulation of immunosuppressive and innate lymphoid cells. Int J Cancer 134:1669-1682

34. Kuchler AM, Pollheimer J, Balogh J, Sponheim J, Manley L, Sorensen DR, De Angelis PM, Scott H, Haraldsen G (2008) Nuclear interleukin-33 is generally expressed in resting endothelium but rapidly lost upon angiogenic or proinflammatory activation. Am J Pathol 173:1229-1242

35. Contasta I, Berghella AM, Pellegrini P, Adorno D (2003) Passage from normal mucosa to adenoma and colon cancer: alteration of normal sCD30 mechanisms regulating TH1/TH2 cell functions. Cancer Biother Radiopharm 18:549-557 
36. Marvie P, Lisbonne M, L'Helgoualc'h A, Rauch M, Turlin B, Preisser L, BourdBoittin K, Theret N, Gascan H, Piquet-Pellorce C, Samson M (2010) Interleukin-33 overexpression is associated with liver fibrosis in mice and humans. J Cell Mol Med 14:1726-1739

37. Meephansan J, Tsuda H, Komine M, Tominaga S, Ohtsuki M (2012) Regulation of IL-33 expression by IFN-gamma and tumor necrosis factor-alpha in normal human epidermal keratinocytes. J Invest Dermatol 132:2593-2600

38. Byrne SN, Beaugie C, O'Sullivan C, Leighton S, Halliday GM (2011) The immunemodulating cytokine and endogenous Alarmin interleukin-33 is upregulated in skin exposed to inflammatory UVB radiation. Am J Pathol 179:211-222

39. Seltmann J, Werfel T, Wittmann M (2013) Evidence for a regulatory loop between IFN-gamma and IL-33 in skin inflammation. Exp Dermatol 22:102-107

40. Gschwantler M, Kriwanek S, Langner E, Goritzer B, Schrutka-Kolbl C, Brownstone E, Feichtinger H, Weiss W (2002) High-grade dysplasia and invasive carcinoma in colorectal adenomas: a multivariate analysis of the impact of adenoma and patient characteristics. Eur J Gastroenterol Hepatol 14:183-188

41. Ajdukovic J, Tonkic A, Salamunic I, Hozo I, Simunic M, Bonacin D (2010) Interleukins IL-33 and IL-17/IL-17A in patients with ulcerative colitis. Hepatogastroenterology 57:1442-1444

42. Seidelin JB, Bjerrum JT, Coskun M, Widjaya B, Vainer B, Nielsen OH (2010) IL-33 is upregulated in colonocytes of ulcerative colitis. Immunol Lett 128:80-85

43. Choi YS, Choi HJ, Min JK, Pyun BJ, Maeng YS, Park H, Kim J, Kim YM, Kwon YG (2009) Interleukin-33 induces angiogenesis and vascular permeability through ST2/TRAF6-mediated endothelial nitric oxide production. Blood 114:3117-3126 


\section{Legends}

Figure 1 legend

Dynamic changes of IL-33 and ST2 transcripts along the colorectal adenoma-carcinoma sequence.

The quantitative real-time PCR results showed that the expression of IL-33 mRNA was significantly increased to a 9 -fold higher level in adenoma tissues (grey bar in Fig. 1A); it was also higher in sporadic CRC tissues (black bar in Fig. 1A) than in normal controls (white bar in Fig. 1A), but significantly lower compared to the adenomas. The expression of ST2 mRNA in adenoma tissues was increased to a $\sim 3$-fold higher level and slightly increased in CRC tissues compared to normal controls (Fig. 1B).

(Y axes in Fig. 1A \& $\mathbf{B}$ are fold changes relative to normal controls; $\mathrm{P}$ values are derived from the Mann-Whitney test) 
Figure 2 legend

Kaplan-Meier curves of overall survival differences among patients with sporadic CRC.

The Kaplan-Meier analysis shows that the expression levels of IL-33 (Fig. 2A) and ST2 (Fig.

2B) do not predicate the overall survival time in patients with sporadic CRC (both P values determined by the log-rank test). 


\section{Figure 3 legend}

Photograph presentations of IL-33 and its receptor, ST2, in the tumor stroma and the adenomatous/cancerous epithelium

Immunohistochemical (IHC) results show that IL-33 immunoreactivity was not observed in the normal epithelium, and a low density of IL-33-positive cells was found in the lamina propria in the control (Fig. 3A). In both the adenoma and CRC, IL-33 immunoreactivity was frequently observed in tumor-associated microvessels (arrow in $3 \mathbf{B} \&$ C) and adenomatous/cancerous epithelium (arrow in Fig. 3B for adenoma and inserted image in 3C for CRC). ST2 immunoreactivity was observed in both the epithelium (arrow head) and

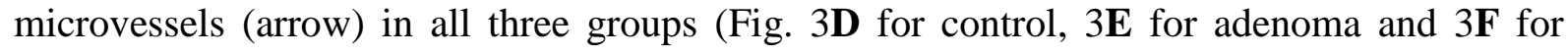
CRC).

(A-E: IHC, counterstained with hematoxylin, original magnification 200x). 
Figure 4 legend

The density grading scores of IL-33 and ST2 positive cells in adenomas and CRC tissues

The semi-quantitative results showed that the density of IL-33-positive cells in the adenomatous epithelium was higher than in the controls (gray bar in Fig. 4A). The density of IL-33-positive cells was also higher in the CRC epithelium (black bar in Fig. 4A) compared to the controls, but lower than the adenomatous epithelium (Fig. 4A). The density of IL-33positive stromal cells was increased in the adenoma stroma, with a smaller increase in magnitude of density in the CRC stroma (Fig. 4B). The density of ST2-positive cells in the adenomatous epithelium was non-statistically increased and was unchanged in the CRC cancerous epithelium (Fig. 4C). The density of ST2-positive cells in the stroma showed a gradually increasing trend from the control to adenoma to $\mathrm{CRC}$, but these differences were not statistically significant (Fig. 4D).

To examine the expression of IL-33/ST2 in endothelial cells, the number of IL-33-positive tumors associated with microvessel density (MVD) and the number of ST2-positive tumors associated with MVD were counted. The results show that the IL-33-positive MVD was significantly increased in the adenoma stroma and non-statistically increased in the CRC tumor stroma compared to normal lamina propria (Fig. 4E). The number of ST2-positive microvessels was greatly increased in both the adenoma stroma and the CRC tumor stroma compared to the normal lamina propria (Fig. 4F).

(HPF: high powder field). 
Figure 5 legend

The phenotypic characterization of IL-33-expressing cells in the adenoma and CRC tumor stromas

Double IHC with IL-33/CD34 and IL-33/SMA-alpha revealed that an increase in IL-33positive cells (red) in the adenoma and CRC tumor stromas was frequently co-localized with CD34-labeled microvessels (blue) (Fig. 5B \& C) and SMA-alpha labeled myofibroblasts (blue) (Fig. 5E \& $\mathbf{F}$ ) compared to the normal lamina propria (Fig. 5A \& D).

(A-F: double IHC, original magnification 400x; counterstaining was not applied) 